Research Article

\title{
Global Structure of Positive Solutions for a Singular Fourth-Order Integral Boundary Value Problem
}

\author{
Wenguo Shen and Tao He \\ Department of Basic Courses, Lanzhou Institute of Technology, Lanzhou 730050, China \\ Correspondence should be addressed to Wenguo Shen; shenwg369@163.com
}

Received 11 November 2013; Revised 14 December 2013; Accepted 16 December 2013; Published 8 January 2014

Academic Editor: Gabriele Bonanno

Copyright (C) 2014 W. Shen and T. He. This is an open access article distributed under the Creative Commons Attribution License, which permits unrestricted use, distribution, and reproduction in any medium, provided the original work is properly cited.

We consider fourth-order boundary value problems $u^{\prime \prime \prime \prime}(t)=\lambda h(t) f(u(t)), 0<t<1, u(0)=\int_{0}^{1} u(s) d \alpha(s), u^{\prime}(0)=u(1)=$ $u^{\prime}(1)=0$, where $\int_{0}^{1} u(s) d \alpha(s)$ is a Stieltjes integral with $\alpha(t)$ being nondecreasing and $\alpha(t)$ being not a constant on $[0,1] ; h(t)$ may be singular at $t=0$ and $t=1, h \in C((0,1),[0, \infty))$ with $h(t) \neq \equiv$ on any subinterval of $(0,1) ; f \in C([0, \infty),[0, \infty))$ and $f(s)>0$ for all $s>0$, and $f_{0}=\infty, f_{\infty}=0, f_{0}=\lim _{s \rightarrow 0^{+}} f(s) / s, f_{\infty}=\lim _{s \rightarrow+\infty} f(s) / s$. We investigate the global structure of positive solutions by using global bifurcation techniques.

\section{Introduction}

Recently, fourth-order boundary value problem

$$
\begin{gathered}
x^{\prime \prime \prime \prime}+k x^{\prime \prime}+l x=\lambda h(t) f(x), \quad 0<t<1, \\
x(0)=x(1)=x^{\prime}(0)=x^{\prime}(1)=0
\end{gathered}
$$

has been investigated by the fixed point theory in cones, see [1-4] $(k=l=0)$. By applying bifurcation techniques, see Rynne [5] $(k=l=0)$, Korman [6] $(k=l=0), \mathrm{Xu}$ and Han [7] $(k=0, l \neq 0)$, Shen $[8,9](k \neq 0, l \neq 0)$, and references therein. However, these papers only studied the nonsingular boundary value problems.

In 2008, Webb et al. [10] studied the existence of multiple positive solutions of nonlinear nonlocal boundary value problems (BVPs) for equations of the form

$$
\begin{gathered}
u^{\prime \prime \prime \prime}(t)=g(t) \hat{f}(t, u(t)), \quad \text { for almost every } t \in(0,1), \\
u(0)=\int_{0}^{1} u(s) d A(s), \quad u^{\prime}(0)=u(1)=u^{\prime}(1)=0,
\end{gathered}
$$

where $g, \widehat{f}$ are continuous and nonnegative functions and $A$ is a function of bounded variation. They treat many boundary conditions appearing in the literature in a unified way.
The main tool they used is the fixed point index theory in cones. In 2009, Ma and An [11] studied the global structure for second-order nonlocal boundary value problem involving Stieltjes integral conditions by applying bifurcation techniques.

Motivated by above papers, in this paper, we will use global bifurcation techniques to study the global structure of positive solutions of the singular problem

$$
\begin{gathered}
u^{\prime \prime \prime \prime}(t)=\lambda h(t) f(u(t)), \quad 0<t<1, \\
u(0)=\int_{0}^{1} u(s) d \alpha(s), \quad u^{\prime}(0)=u(1)=u^{\prime}(1)=0,
\end{gathered}
$$

where $h(t)$ may be singular at $t=0$ and $t=1$, and $\lambda \in(0, \infty)$ is a parameter.

In order to prove our main result, let us make the assumptions as follows:

(A1) $\alpha:[0,1] \rightarrow \mathbb{R}$ is nondecreasing and $\alpha(t)$ is not a constant on $[0,1], \int_{0}^{1} k(t, s) d \alpha(t) \geq 0$ for $s \in[0,1]$, and $0 \leq a<1$ with $a:=\int_{0}^{1} \gamma(t) d \alpha(t), \gamma(t)=(t-1)^{2}$ $(2 t+1)$

(A2) $h \in C((0,1),[0, \infty))$ with $h(t) \not \equiv 0$ on any subinterval of $(0,1)$, and $0<\int_{0}^{1} h(s) d s<\infty$; 
(A3) $f \in C([0, \infty),[0, \infty))$ satisfies $f(s)>0$ for all $s>0$;

(A4) $f_{0}=\lim _{s \rightarrow 0^{+}}(f(s) / s)=\infty$;

(A5) $f_{\infty}=\lim _{s \rightarrow+\infty}(f(s) / s)=0$.

Remark 1. For other results on the existence and multiplicity of positive solutions and nodal solutions for the boundary value problems of fourth-order ordinary differential equations based on bifurcation techniques, see $\mathrm{Ma}$ et al. [12-15] and Bai and Wang [16] and their references.

The rest of the paper is arranged as follows: In Section 2, we state some properties of superior limit of certain infinity collection of connected sets. In Section 3, we will give some preliminary results. In Section 4, we state and prove our main results.

\section{Superior Limit and Component}

In order to treat the case $f_{0}=\infty, f_{\infty}=0$, we will need the following definition and lemmas.

Definition 2 (see [17]). Let $X$ be a Banach space and let $\left\{C_{n} \mid\right.$ $n=1,2, \ldots\}$ be a family of subsets of $X$. Then the superior limit $\mathscr{D}$ of $\left\{C_{n}\right\}$ is defined by

$$
\begin{aligned}
\mathscr{D} & :=\limsup _{n \rightarrow \infty} C_{n} \\
& =\left\{x \in X \mid \exists\left\{n_{i}\right\} \subset \mathbb{N}, x_{n_{i}} \in C_{n_{i}} \text {, such that } x_{n_{i}} \longrightarrow x\right\} .
\end{aligned}
$$

Lemma 3 (see [17]). Each connected subset of metric space $X$ is contained in a component, and each connected component of $X$ is closed.

Lemma 4 (see [11]). Let $X$ be a Banach space and let $\left\{C_{n} \mid n=\right.$ $1,2, \ldots\}$ be a family of closed connected subsets of $X$. Assume that

(i) there exist $z_{n} \in C_{n}, n=1,2, \ldots$, and $z^{*} \in X$, such that $z_{n} \rightarrow z^{*}$

(ii) $r_{n}=\sup \left\{\|x\| \mid x \in C_{n}\right\}=\infty$;

(iii) for all $R>0,\left(\cup_{n=1}^{\infty} C_{n}\right) \cap B_{R}$ is a relative compact set of $X$, where

$$
B_{R}=\{x \in X \mid\|x\| \leq R\} .
$$

Then there exists an unbounded connected component $C$ in $\mathbb{D}$ and $z^{*} \in C$.

\section{Preliminaries}

We consider the problem as follows:

$$
\begin{gathered}
u^{\prime \prime \prime \prime}(t)=y(t), \quad 0<t<1, \\
u(0)=\int_{0}^{1} u(s) d \alpha(s), \quad u^{\prime}(0)=u(1)=u^{\prime}(1)=0 .
\end{gathered}
$$

Lemma 5. For any $y \in C[0,1]$, the problem (6) has a unique solution

$$
u(t)=\int_{0}^{1} K(t, s) y(s) d s
$$

where

$$
K(t, s)=\frac{\gamma(t)}{1-a} \int_{0}^{1} k(t, s) d \alpha(t)+k(t, s)
$$

$k(t, s)=\frac{1}{6} \begin{cases}t^{2}(1-s)^{2}[(s-t)+2(1-t) s], & 0 \leq t \leq s \leq 1 \\ s^{2}(1-t)^{2}[(t-s)+2(1-s) t], & 0 \leq s \leq t \leq 1\end{cases}$

$a=\int_{0}^{1} \gamma(t) d \alpha(t), \gamma(t)=(t-1)^{2}(2 t+1)$.

Proof. By [10], the problem (6) can be equivalently written as

$$
u(t)=\gamma(t) \int_{0}^{1} u(s) d \alpha(s)+\int_{0}^{1} k(t, s) y(s) d s .
$$

Applying $\alpha$ to both sides of (10), we obtain

$$
\begin{aligned}
& \int_{0}^{1} u(t) d \alpha(t) \\
& \quad=\int_{0}^{1}\left[\gamma(t) \int_{0}^{1} u(s) d \alpha(s)\right] d \alpha(t) \\
& \quad+\int_{0}^{1}\left[\int_{0}^{1} k(t, s) y(s) d s\right] d \alpha(t) .
\end{aligned}
$$

Thus, we have

$$
\begin{aligned}
\int_{0}^{1} u(t) d \alpha(t)= & \int_{0}^{1} \gamma(t) d \alpha(t) \cdot \int_{0}^{1} u(s) d \alpha(s) \\
& +\int_{0}^{1}\left[\int_{0}^{1} k(t, s) d \alpha(t)\right] y(s) d s .
\end{aligned}
$$

Furthermore, it follows that

$$
\begin{aligned}
& \int_{0}^{1} u(s) d \alpha(s) \\
& \quad=\frac{1}{1-\int_{0}^{1} \gamma(t) d \alpha(t)} \int_{0}^{1}\left[\int_{0}^{1} k(t, s) d \alpha(t)\right] y(s) d s .
\end{aligned}
$$

So, we obtain

$$
u(t)=\int_{0}^{1}\left[\frac{\gamma(t)}{1-a} \int_{0}^{1} k(t, s) d \alpha(t)+k(t, s)\right] y(s) d s .
$$

Lemma 6 (see [2-4]). Green's function $k(t, s)$ defined by (9) satisfies the following:

(i) $k(t, s) \geq 0$ is continuous for all $t, s \in[0,1]$;

(ii) $c(t) k(\tau(s), s) \leq k(t, s) \leq k(\tau(s), s)$, for all $t, s \in[0,1]$, and for any $\delta \in(0,1 / 2)$ and $t \in[\delta, 1-\delta]$, such that

$$
k(t, s) \geq \frac{2}{3} \delta^{2} k(\tau(s), s), \quad \forall s \in[0,1],
$$


where

$$
\begin{gathered}
\tau(s)= \begin{cases}\frac{1}{3-2 s}, & 0 \leq s \leq \frac{1}{2}, \\
\frac{2 s}{1+2 s}, & \frac{1}{2} \leq s \leq 1,\end{cases} \\
k(\tau(s), s)=\max _{t \in[0,1]} k(t, s)= \begin{cases}\frac{2 s^{2}(1-s)^{3}}{3(3-2 s)^{2}}, & 0 \leq s \leq \frac{1}{2}, \\
\frac{2 s^{3}(1-s)^{2}}{3(1+2 s)^{2}}, & \frac{1}{2} \leq s \leq 1,\end{cases} \\
c(t)=\frac{2}{3} \min \left\{t^{2},(1-t)^{2}\right\}, \quad t \in[0,1], \\
\min _{t \in[\delta, 1-\delta]} c(t)=\frac{2}{3} \delta^{2} .
\end{gathered}
$$

Lemma 7. Green's function $K(t, s)$ defined by (8) satisfies the following:

(i) $K(t, s) \geq 0$ is continuous for all $t, s \in[0,1]$;

(ii) $K(t, s) \leq K(s)$, for all $t, s \in[0,1]$, and for any $\delta \in$ $(0,1 / 2)$, there exists a constant $\gamma_{\delta}>0$, for any $t \in$ $[\delta, 1-\delta]$, such that

$K(t, s) \geq \gamma_{\delta} K(s), \quad \forall s \in[0,1]$,

$$
\begin{gathered}
K(s)=\frac{1-a+\alpha(1)-\alpha(0)}{1-a} \cdot k(\tau(s), s), \\
\gamma_{\delta}=\frac{2}{3} \delta^{2} \cdot \frac{1-a+\delta^{2}(3-2 \delta)(\alpha(1)-\alpha(0))}{1-a+\alpha(1)-\alpha(0)},
\end{gathered}
$$

where $k(t, s)$ is defined by $(9), \max _{t \in[0,1]} \gamma(t)=1$, $\min _{t \in[\delta, 1-\delta]} \gamma(t)=\delta^{2}(3-2 \delta)$.

Proof. (i) From Lemma 6 (i), we get the proof of Lemma 7 (i) immediately.

(ii) By Lemma 6 (ii), we get

$$
\begin{array}{r}
K(t, s) \leq \frac{1}{1-a} \int_{0}^{1} k(\tau(s), s) d \alpha(t)+k(\tau(s), s) \\
\leq \frac{1-a+\alpha(1)-\alpha(0)}{1-a} \cdot k(\tau(s), s)=K(s), \\
\forall t, s \in[0,1] .
\end{array}
$$

By Lemma 6 (ii), for any $\delta \in(0,1 / 2)$ and $t \in[\delta, 1-\delta], s \in$ $[0,1]$, we obtain

$$
\begin{aligned}
K(t, s) \geq & \frac{\delta^{2}(3-2 \delta)}{1-a} \int_{0}^{1} \frac{2}{3} \delta^{2} k(\tau(s), s) d \alpha(t) \\
& +\frac{2}{3} \delta^{2} k(\tau(s), s) \\
\geq & \frac{2}{3} \delta^{2} \cdot \frac{1-a+\delta^{2}(3-2 \delta)(\alpha(1)-\alpha(0))}{1-a} \\
& \cdot k(\tau(s), s)=\gamma_{\delta} K(s), \quad \forall s \in[0,1] .
\end{aligned}
$$

Lemma 8. For $y \in C[0,1]$ and $y \geq 0$, the unique solution of the problem (6) satisfies the following:

(i) $u(t) \geq 0$, for all $t \in[0,1]$;

(ii) $\min _{t \in[\delta, 1-\delta]} u(t) \geq \gamma_{\delta}\|u\|_{\infty}$,

where $\gamma_{\delta}$ is defined by Lemma 7 (ii), $\|u\|_{\infty}=\max _{t \in[0,1]}|u|$.

Proof. (i) From Lemma 7 (i), we get the proof of Lemma 8 (i) immediately.

(ii) From (7) and Lemma 7, we have

$$
\begin{aligned}
\min _{t \in[\delta, 1-\delta]} u(t) & =\min _{t \in[\delta, 1-\delta]} \int_{0}^{1} K(t, s) y(s) d s \\
& \geq \int_{0}^{1} \min _{t \in[\delta, 1-\delta]} K(t, s) y(s) d s \\
& \geq \gamma_{\delta} \int_{0}^{1} K(s) y(s) d s \\
& \geq \gamma_{\delta} \int_{0}^{1} \max _{t \in[0,1]} K(t, s) y(s) d s \\
& \geq \gamma_{\delta} \max _{t \in[0,1]} \int_{0}^{1} K(t, s) y(s) d s=\gamma_{\delta}\|u\|_{\infty} .
\end{aligned}
$$

Therefore, the proof of Lemma 8 is complete.

Let $Y=C[0,1]$ be the Banach space with the norm $\|u\|_{\infty}=\max _{t \in[0,1]}|u|$.

Let $E=\left\{u \in C^{2}[0,1] \mid u(0)=\int_{0}^{1} u(s) d \alpha(s), u^{\prime}(0)=\right.$ $\left.u(1)=u^{\prime}(1)=0\right\}$ with the norm

$$
\|u\|_{E}=\max \left\{\|u\|_{\infty},\left\|u^{\prime}\right\|_{\infty},\left\|u^{\prime \prime}\right\|_{\infty}\right\} .
$$

Let

$$
\begin{gathered}
P=\{u \in C[0,1] \mid u(t) \geq 0, t \in[0,1], \\
\left.\min _{t \in[\delta, 1-\delta]} u(t) \geq \gamma_{\delta}\|u\|_{\infty}\right\},
\end{gathered}
$$

and for $r>0$, let $\Omega_{r}=\left\{u \in P \mid\|u\|_{E}<r\right\}$.

In order to use bifurcation technique to study the problem (3), we consider the linear eigenvalue problem

$$
u^{\prime \prime \prime \prime}(t)=\lambda h(t) u(t), \quad 0<t<1,
$$

$u(0)=\int_{0}^{1} u(s) d \alpha(s), \quad u^{\prime}(0)=u(1)=u^{\prime}(1)=0$.

Let

$$
\begin{gathered}
L_{\lambda} u(t)=\lambda \int_{0}^{1} K(t, s) h(s) u(s) d s, \quad t \in[0,1], \\
T_{\lambda} u(t)=\lambda \int_{0}^{1} K(t, s) h(s) f(u(s)) d s, \quad t \in[0,1] .
\end{gathered}
$$

By [18], it is easy to show the following lemma. 
Lemma 9. Assume that (A1)-(A3) hold the following.

$L_{\lambda}: P \rightarrow P$ is a completely continuous linear operator and $L_{\lambda}(P) \subset P$, and the fixed points of the operator $L_{\lambda}$ in $P$ are the positive solutions of the BVP (23).

$T_{\lambda}: P \rightarrow P$ is a completely continuous operator and $T_{\lambda}(P) \subset P$, and the fixed points of the operator $T_{\lambda}$ in $P$ are the positive solutions of the BVP (3).

By virtue of Krein-Rutman theorem (Theorem 2.5 in [19]), one has (see [18] or [20]) the following lemma.

Lemma 10. Suppose that $L_{\lambda}: C[0,1] \rightarrow C[0,1]$ is a completely continuous linear operator and $L_{\lambda}(P) \subset P$. If there exist $\psi \in C[0,1] \backslash(-P)$ and a constant $c>0$ such that $c L_{\lambda} \psi \geq \psi$, then the spectral radius $r\left(L_{\lambda}\right) \neq 0$ and $L_{\lambda}$ has a positive eigenfunction $\phi_{1}$ corresponding to its first eigenvalue $\lambda_{1}=1 / r\left(L_{\lambda}\right)$, that is, $\phi_{1}=\lambda_{1} L_{\lambda} \phi_{1}$.

Lemma 11. Suppose (A1) and (A2) are satisfied, then for the operator $L_{\lambda}$ defined by (24), the spectral radius $r\left(L_{\lambda}\right) \neq 0$ and $L_{\lambda}$ has a positive eigenfunction $\phi_{1} \in$ int $P$ corresponding to its first eigenvalue $\lambda_{1}=1 / r\left(L_{\lambda}\right)$.

Proof. It is easy to see that there is $t_{1} \in(0,1)$ such that $K\left(t_{1}, t_{1}\right) h\left(t_{1}\right)>0$. Thus there exists $[\alpha, \beta] \subset(0,1)$ such that $t_{1} \in(\alpha, \beta)$ and $K(t, s) h(s)>0$, for all $t, s \in[\alpha, \beta]$. Take $\psi \in C[0,1]$ such that $\psi(t) \geq 0$, for all $t \in[0,1], \psi\left(t_{1}\right)>0$, and $\psi(t)=0$, for all $t \notin[\alpha, \beta]$. Then for $t \in[\alpha, \beta]$,

$$
\begin{aligned}
\left(L_{\lambda} \psi\right)(t) & =\lambda \int_{0}^{1} K(t, s) h(s) \psi(s) d s \\
& \geq \lambda \int_{\alpha}^{\beta} K(t, s) h(s) \psi(s) d s>0 .
\end{aligned}
$$

So there exists a constant $c>0$ such that $c L_{\lambda} \psi \geq \psi$, for all $t \in$ $[0,1]$. From Lemma 10, we know that the spectral radius $r\left(L_{\lambda}\right) \neq 0$ and $L_{\lambda}$ has a positive eigenfunction corresponding to its first eigenvalue $\lambda_{1}=1 / r\left(L_{\lambda}\right)$.

Lemma 12. Let (A1)-(A3) hold. The solution $u(t)$ of the problem (3) satisfies

$$
\|u\|_{\infty} \leq\left\|u^{\prime}\right\|_{\infty} \leq\left\|u^{\prime \prime}\right\|_{\infty} \leq\left\|u^{\prime \prime \prime}\right\|_{\infty}
$$

Proof. From $u^{\prime}(0)=u^{\prime}(1)=0$, there exists $\xi \in(0,1)$, such that $u^{\prime \prime}(\xi)=0$. Using a similar proof of $(10)$ in [21, page 212], it is easy to show that

$$
\begin{aligned}
|u(t)| & =\left|\int_{t}^{1} u^{\prime}(s) d s-u(1)\right|=\left|\int_{t}^{1} u^{\prime}(s) d s\right| \\
& \leq \int_{t}^{1}\left|u^{\prime}(s)\right| d s \leq \int_{0}^{1}\left|u^{\prime}(s)\right| d s, \\
\left|u^{\prime}(t)\right| & =\left|u^{\prime}(0)+\int_{0}^{t} u^{\prime \prime}(s) d s\right| \\
& =\left|\int_{0}^{t} u^{\prime \prime}(s) d s\right| \leq \int_{0}^{1}\left|u^{\prime \prime}(s)\right| d s,
\end{aligned}
$$

$$
\begin{aligned}
\left|u^{\prime \prime}(t)\right| & =\left|u^{\prime \prime}(\xi)+\int_{\xi}^{t} u^{\prime \prime \prime}(s) d s\right| \\
& =\left|\int_{\xi}^{t} u^{\prime \prime \prime}(s) d s\right| \leq \int_{0}^{1}\left|u^{\prime \prime \prime}(s)\right| d s .
\end{aligned}
$$

Furthermore, we obtain

$$
\|u\|_{\infty} \leq\left\|u^{\prime}\right\|_{\infty} \leq\left\|u^{\prime \prime}\right\|_{\infty} \leq\left\|u^{\prime \prime \prime}\right\|_{\infty} .
$$

Lemma 13. Let (A1)-(A3) hold. Assume that $\left\{\left(\mu_{k}, u_{k}\right)\right\} \quad \subset$ $(0, \infty) \times P$ is a sequence of positive solutions of (3). Assume that $\left\|\mu_{k}\right\| \leq c_{0}$ for some constant $c_{0}>0$, and

$$
\lim _{k \rightarrow \infty}\left\|u_{k}\right\|_{E}=\infty
$$

Then

$$
\lim _{k \rightarrow \infty}\left\|u_{k}\right\|_{\infty}=\infty
$$

Proof. Assume on the contrary that

$$
\left\|u_{k}\right\|_{\infty} \leq M_{0}
$$

for some constant $M_{0}>0$.

Since $\left(\mu_{k}, u_{k}\right)$ is a solution of the problem (3), we have

$$
u_{k}(t)=\mu_{k} \int_{0}^{1} K(t, s) h(s) f\left(u_{k}(s)\right) d s, \quad t \in[0,1] .
$$

Thus,

$$
\begin{aligned}
u_{k}^{\prime \prime \prime}(t) & =\mu_{k} \int_{0}^{1} \frac{\partial^{3}}{\partial t^{3}} K(t, s) \cdot h(s) f\left(u_{k}(s)\right) d s \\
\left|\frac{\partial^{3}}{\partial t^{3}} K(t, s)\right| & =\left|\frac{12}{1-a} \int_{0}^{1} k(t, s) d \alpha(t)+\frac{\partial^{3}}{\partial t^{3}} k(t, s)\right| \\
& \leq\left|\frac{12(\alpha(1)-\alpha(0))}{1-a} k(\tau(s), s)+\frac{\partial^{3}}{\partial t^{3}} k(t, s)\right| \\
& \leq \frac{\alpha(1)-\alpha(0)}{16(1-a)}+5:=M_{1},
\end{aligned}
$$

where $0 \leq|k(\tau(s), s)| \leq 1 / 192, \sup _{0 \leq t, s \leq 1, t \neq s}\left|\left(\partial^{3} / \partial t^{3}\right) k(t, s)\right| \leq$ 5 (see [3]).

Furthermore, it follows that

$$
\left\|u_{k}^{\prime \prime \prime}\right\|_{\infty} \leq c_{0} M_{1} B_{0} \int_{0}^{1} h(s) d s,
$$

where $B_{0}=\max _{s \in\left[0, M_{0}\right]}\{f(s)\}$, together with $\left(A_{2}\right)$, which implies that $\left\|u_{k}^{\prime \prime \prime}\right\|_{\infty}$ is bounded whenever $\left\|u_{k}\right\|_{\infty}$ is bounded.

Together with Lemma 12, we obtain

$$
\left\|u_{k}\right\|_{E} \leq M_{2}
$$

for some constant $M_{2}>0$. This is a contradiction. 


\section{Main Results}

Let $\Sigma$ be the closure of the set of positive solutions of (3) in $[0, \infty) \times E$. The main results of this paper are the following.

Theorem 14. Let (A1)-(A5) hold, then (3) has at least one solution for any $\lambda \in(0, \infty)$.

Let $L: D(L) \subset E \rightarrow E$ be an operator defined by

$$
L u=u^{\prime \prime \prime \prime}, \quad u \in D(L),
$$

with

$$
\begin{gathered}
D(L)=\left\{u \in C^{4}[0,1] \mid u(0)=\int_{0}^{1} u(s) d \alpha(s),\right. \\
\left.u^{\prime}(0)=u(1)=u^{\prime}(1)=0\right\} .
\end{gathered}
$$

Then $L$ is a closed operator and $L^{-1}: Y \rightarrow E$ is completely continuous.

For each $n \in \mathbb{N}$, define $f^{[n]}(s):[0, \infty) \rightarrow[0, \infty)$ by

$$
f^{[n]}(s)= \begin{cases}f(s), & s \in\left(\frac{1}{n}, \infty\right), \\ n f\left(\frac{1}{n}\right) s, & s \in\left[0, \frac{1}{n}\right] .\end{cases}
$$

Then $f^{[n]} \in C([0, \infty),[0, \infty))$ with

$$
f^{[n]}(s)>0, \quad \forall s \in(0, \infty), \quad\left(f^{[n]}\right)_{0}=n f\left(\frac{1}{n}\right) .
$$

By (A4), it follows that

$$
\lim _{n \rightarrow \infty}\left(f^{[n]}\right)_{0}=\infty .
$$

To apply the global bifurcation theorem, one extends $f$ to an odd function $g: \mathbb{R} \rightarrow \mathbb{R}$ by

$$
g(s)= \begin{cases}f(s), & s \geq 0 \\ -f(-s), & s<0\end{cases}
$$

Similarly one may extend $f^{[n]}$ to an odd function $g^{[n]}: \mathbb{R} \rightarrow$ $\mathbb{R}$ for each $n \in \mathbb{N}$.

Now let one consider the auxiliary family of the equations

$$
\begin{gathered}
u^{\prime \prime \prime \prime}(t)=\lambda h(t) g^{[n]}(u(t)), \quad 0<t<1, \\
u(0)=\int_{0}^{1} u(s) d \alpha(s), \quad u^{\prime}(0)=u(1)=u^{\prime}(1)=0 .
\end{gathered}
$$

Let $\zeta^{[n]} \in C(\mathbb{R})$ be such that

$$
g^{[n]}(u)=\left(g^{[n]}\right)_{0} u+\zeta^{[n]}(u)=n f\left(\frac{1}{n}\right) u+\zeta^{[n]}(u) .
$$

Then

$$
\lim _{|s| \rightarrow 0} \frac{\zeta^{[n]}(s)}{s}=0 .
$$

Let one consider

$$
L u=\lambda h(t)\left(g^{[n]}\right)_{0} u+\lambda h(t) \zeta^{[n]}(u)
$$

as a bifurcation problem from the trivial solution $u \equiv 0$.
From Lemma 5, (46) can be converted to the equivalent equation

$$
\begin{aligned}
u(t) & =\int_{0}^{1} K(t, s)\left[\lambda h(s)\left(g^{[n]}\right)_{0} u(s)+\lambda h(s) \zeta^{[n]}(u(s))\right] d s \\
& :=\lambda L^{-1}\left[h(\cdot)\left(g^{[n]}\right)_{0} u(\cdot)\right](t)+\lambda L^{-1}\left[h(\cdot) \zeta^{[n]}(u(\cdot))\right](t) .
\end{aligned}
$$

Further one has that

$$
\left\|L^{-1}\left[h(\cdot) \zeta^{[n]}(u(\cdot))\right]\right\|_{E}=o\left(\|u\|_{E}\right), \quad \text { as }\|u\|_{E} \longrightarrow 0 .
$$

Indeed, (8) implies that, for all $(t, s) \in[0,1] \times[0,1]$,

$$
\begin{aligned}
\left|\frac{\partial}{\partial t} K(t, s)\right| & =\left|\frac{6 t(t-1)}{1-a} \int_{0}^{1} k(t, s) d \alpha(t)+\frac{\partial}{\partial t} k(t, s)\right| \\
& \leq \frac{\alpha(1)-\alpha(0)}{128(1-a)}+3, \\
\left|\frac{\partial^{2}}{\partial t^{2}} K(t, s)\right| & =\left|\frac{6(2 t-1)}{1-a} \int_{0}^{1} k(t, s) d \alpha(t)+\frac{\partial^{2}}{\partial t^{2}} k(t, s)\right| \\
& \leq \frac{\alpha(1)-\alpha(0)}{32(1-a)}+8,
\end{aligned}
$$

where $0 \leq|k(\tau(s), s)| \leq 1 / 192, \max _{0 \leq t, s \leq 1}|(\partial / \partial t) k(t, s)| \leq 3$, $\max _{0 \leq t, s \leq 1}\left|\left(\partial^{2} / \partial t^{2}\right) k(t, s)\right| \leq 8$ (see [3]).

So, the compactness of $L^{-1}$ together with (45) and (A2) imply that

$$
\begin{gathered}
\left\|\left(L^{-1}\left[h(\cdot) \zeta^{[n]}(u(\cdot))\right]\right)^{\prime}\right\|_{\infty}=o\left(\|u\|_{\infty}\right), \\
\left\|\left(L^{-1}\left[h(\cdot) \zeta^{[n]}(u(\cdot))\right]\right)^{\prime \prime}\right\|_{\infty}=o\left(\|u\|_{\infty}\right),
\end{gathered}
$$

and consequently

$$
\begin{gathered}
\left\|\left(L^{-1}\left[h(\cdot) \zeta^{[n]}(u(\cdot))\right]\right)^{\prime}\right\|_{\infty}=o\left(\|u\|_{E}\right), \\
\left\|\left(L^{-1}\left[h(\cdot) \zeta^{[n]}(u(\cdot))\right]\right)^{\prime \prime}\right\|_{\infty}=o\left(\|u\|_{E}\right) .
\end{gathered}
$$

Let $S_{0}^{+}=\{u \in E \mid u(t)>0$, for all $t \in[0,1]\}, S_{0}^{-}=$ $\{u \in E \mid u(t)<0$, for all $t \in[0,1]\}$, then $S_{0}^{+} \cap S_{0}^{-}=\emptyset$. Let $S_{0}=S_{0}^{+} \cup S_{0}^{-}, \Phi_{0}^{ \pm}=R \times S_{0}^{ \pm}$.

By Lemma 11 and the fact $\left(g^{[n]}\right)_{0}>0$, the global bifurcation result (see Rabinowitz [22]) for (46) can be stated as follows: there exists a continuum $C_{+}^{[n]}(\subset \quad[0, \infty) \times P)$ of positive solutions of (46) joining $\left(\lambda_{1} /\left(g^{[n]}\right)_{0}, 0\right)$ to infinity in $[0, \infty) \times P$. Moreover, $\left(\lambda_{1} /\left(g^{[n]}\right)_{0}, \theta\right)$ is the only positive bifurcation point of (46) lying on trivial solutions line $u \equiv \theta$. Moreover, $C_{+}^{[n]} ।$ $\left\{\left(\lambda_{1} /\left(g^{[n]}\right)_{0}, 0\right)\right\} \subset \Phi_{0}^{+}$.

Since

$$
\lim _{n \rightarrow \infty} \frac{\lambda_{1}}{\left(g^{[n]}\right)_{0}}=\lim _{n \rightarrow \infty} \frac{\lambda_{1}}{n f(1 / n)}=0
$$


condition (i) in Lemma 4 is satisfied with $z^{*}=(0,0)$. Obviously

$$
r_{n}=\sup \left\{\lambda+\|u\| \mid(\lambda, u) \in C_{+}^{[n]}\right\}=\infty,
$$

and accordingly (ii) in Lemma 4 holds. (iii) in Lemma 4 can be deduced directly from the Arzela-Ascoli theorem and the definition of $g^{[n]}$. Therefore, the superior limit of $\left\{C_{+}^{[n]}\right\}, \mathscr{D}$, contained an unbounded connected component $C$ with $(0,0) \epsilon$ C. Since $C_{+}^{[n]} \subset \Phi^{+}$, one concludes $C \subset \Phi^{+}$. Moreover, $C \subset \Sigma$ by (3).

Proof of Theorem 14. We firstly prove

$$
\operatorname{Proj}_{R} C=(0, \infty) .
$$

Assume on the contrary that

$$
\sup \{\lambda \mid(\lambda, u) \in C\}<\infty .
$$

Then there exists a sequence $\left(\mu_{k}, u_{k}\right) \in C$ such that

$$
\lim _{k \rightarrow \infty}\left\|u_{k}\right\|_{E}=\infty, \quad \mu_{k} \leq c_{0},
$$

for some positive constant $c_{0}$ with doing not depend on $k$. From Lemma 13, we have

$$
\lim _{k \rightarrow \infty}\left\|u_{k}\right\|_{\infty}=\infty .
$$

This together with the fact

$$
\min _{t \in[\delta, 1-\delta]} u_{k}(t) \geq \gamma_{\delta}\left\|u_{k}\right\|_{\infty}, \quad \forall \delta \in\left(0, \frac{1}{2}\right)
$$

imply that for arbitrary $\delta \in(0,1 / 2)$

$$
\lim _{k \rightarrow \infty} u_{k}(t)=\infty, \quad \text { uniformly for } t \in[\delta, 1-\delta] .
$$

Since $\left(\mu_{k}, u_{k}\right) \in C$, we have that

$$
\begin{gathered}
u_{k}^{\prime \prime \prime \prime}=\mu_{k} h(t) f\left(u_{k}\right), \quad 0<t<1, \\
u_{k}(0)=\int_{0}^{1} u_{k}(s) d \alpha(s), \quad u_{k}^{\prime}(0)=u_{k}(1)=u_{k}^{\prime}(1)=0 .
\end{gathered}
$$

Set $v_{k}(t)=u_{k}(t) /\left\|u_{k}\right\|_{\infty}$. Then

$$
\begin{gathered}
\left\|v_{k}(t)\right\|_{\infty}=1, \\
v_{k}^{\prime \prime \prime \prime}(t)=\mu_{k} h(t) \frac{f\left(u_{k}(t)\right)}{u_{k}(t)} v_{k}(t), \quad 0<t<1, \\
v_{k}(0)=\int_{0}^{1} y(s) d \alpha(s), \quad v_{k}^{\prime}(0)=v_{k}(1)=v_{k}^{\prime}(1)=0 .
\end{gathered}
$$

Now, choosing a subsequence and relabeling if necessary, it follows that there exists $\left(\mu_{*}, v_{*}\right) \in\left[0, c_{0}\right] \times E$ with

$$
\left\|v_{*}\right\|_{\infty}=1
$$

such that

$$
\lim _{k \rightarrow \infty}\left(\mu_{k}, v_{k}\right)=\left(\mu_{*}, v_{*}\right), \quad \text { in } \mathbb{R} \times Y .
$$

By (A3), let

$$
\bar{f}(u)=\max _{0 \leq s \leq u} f(s) .
$$

Then $\bar{f}$ is nondecreasing and

$$
\lim _{u \rightarrow+\infty} \frac{\bar{f}(u)}{u}=0 .
$$

Further it follows from (66) that

$$
\frac{f(u)}{\|u\|_{\infty}} \leq \frac{\bar{f}(u)}{\|u\|_{\infty}} \leq \frac{\bar{f}\left(\|u\|_{\infty}\right)}{\|u\|_{\infty}} \longrightarrow 0, \quad\|u\|_{\infty} \longrightarrow+\infty .
$$

Thus,

$$
\lim _{k \rightarrow \infty} \frac{f\left(u_{k}\right)}{u_{k}}=0 .
$$

Notice that (62) is equivalent to

$$
v_{k}(t)=\mu_{k} \int_{0}^{1} K(t, s) h(s) \frac{f\left(u_{k}(s)\right)}{u_{k}(s)} v_{k}(s) d s, \quad t \in[0,1] .
$$

Furthermore, by (59), (68), and (69), together with the Lebesgue dominated convergence theorem, it follows that

$$
v_{*}(t)=\mu_{k} \int_{0}^{1} K(t, s) h(s) \cdot 0 \cdot v_{*}(s) d s, \quad t \in[0,1] .
$$

It follows that

$$
v_{*}(t) \equiv 0 .
$$

This contradicts (63). Therefore

$$
\sup \left\{\lambda \mid(\lambda, u) \in C_{+}^{[n]}\right\}=\infty .
$$

Noticing that $\lambda=0$ is the only solution of the problem (3), thus

$$
\operatorname{Proj}_{R} C=(0, \infty) .
$$

Furthermore, it follows the proof of Theorem 14.

\section{Conflict of Interests}

The authors declare that there is no conflict of interests regarding the publication of this paper.

\section{Acknowledgments}

Thanks are given to the anonymous referee for his/her valuable suggestions. The authors were supported by the NSFC (no. 11261052) and the Scientific Research Foundation of the Education department of Gansu Province (no. 1114$04)$. 


\section{References}

[1] R. P. Agarwal and Y. M. Chow, "Iterative methods for a fourth order boundary value problem," Journal of Computational and Applied Mathematics, vol. 10, no. 2, pp. 203-217, 1984.

[2] R. Ma and H. P. Wu, "Positive solutions of a fourth-order twopoint boundary value problem," Acta Mathematica Scientia A, vol. 22, no. 2, pp. 244-249, 2002.

[3] Q. Yao, "Multiple positive solutions to a singular beam equation fixed at both ends," Acta Mathematica Scientia A, vol. 28, no. 4, pp. 768-778, 2008.

[4] Q. Yao, "Positive solutions for eigenvalue problems of fourthorder elastic beam equations," Applied Mathematics Letters, vol. 17, no. 2, pp. 237-243, 2004.

[5] B. P. Rynne, "Infinitely many solutions of superlinear fourth order boundary value problems," Topological Methods in Nonlinear Analysis, vol. 19, no. 2, pp. 303-312, 2002.

[6] P. Korman, "Uniqueness and exact multiplicity of solutions for a class of fourth-order semilinear problems," Proceedings of the Royal Society of Edinburgh A, vol. 134, no. 1, pp. 179-190, 2004.

[7] J. Xu and X. Han, "Nodal solutions for a fourth-order two-Ooint boundary value problem," Boundary Value Problem, vol. 2010, Article ID 570932, 11 pages, 2010.

[8] W. G. Shen, "Existence of nodal solutions of a nonlinear fourthorder two-point boundary value problem," Boundary Value Problems, vol. 2012, p. 31, 2012.

[9] W. G. Shen, "Global structure of nodal solutions for a fourthorder two-point boundary value problem," Applied Mathematics and Computation, vol. 219, no. 1, pp. 88-98, 2012.

[10] J. R. L. Webb, G. Infante, and D. Franco, "Positive solutions of nonlinear fourth-order boundary-value problems with local and non-local boundary conditions," Proceedings of the Royal Society of Edinburgh Section A, vol. 138, no. 2, pp. 427-446, 2008.

[11] R. Ma and Y. An, "Global structure of positive solutions for superlinear second order m-point boundary value problems," Topological Methods in Nonlinear Analysis, vol. 34, no. 2, pp. 279-290, 2009.

[12] R. Ma, "Nodal solutions for a fourth-order two-point boundary value problem," Journal of Mathematical Analysis and Applications, vol. 314, no. 1, pp. 254-265, 2006.

[13] R. Ma, "Nodal solutions of boundary value problems of fourthorder ordinary differential equations," Journal of Mathematical Analysis and Applications, vol. 319, no. 2, pp. 424-434, 2006.

[14] R. Ma and J. Xu, "Bifurcation from interval and positive solutions of a nonlinear fourth-order boundary value problem," Nonlinear Analysis, Theory, Methods and Applications, vol. 72, no. 1, pp. 113-122, 2010.

[15] R. Ma, "Existence of positive solutions of a fourth-order boundary value problem," Applied Mathematics and Computation, vol. 168, no. 2, pp. 1219-1231, 2005.

[16] Z. Bai and H. Wang, "On positive solutions of some nonlinear fourth-order beam equations," Journal of Mathematical Analysis and Applications, vol. 270, no. 2, pp. 347-368, 2002.

[17] G. T. Whyburn, Topological Analysis, Princeton Mathematical Series. No. 23, Princeton University Press, Princeton, NJ, USA, 1958.

[18] G. W. Zhang and J. X. Sun, "Positive solutions of m-point boundary value problems," Journal of Mathematical Analysis and Applications, vol. 291, no. 2, pp. 406-418, 2004.

[19] M. A. Krasnoselskii, Positive Solutions of Operator Equations, P. Noordhoff Limited, Groningen, The Netherlands, 1964.
[20] D. J. Guo and J. X. Sun, Nonlinear Integral Equations, Shandong Science and Technology Press, Jinan, China, 1987.

[21] Y. J. Liu and W. G. Ge, "Positive solutions of two-point boundary value problems for $2 \mathrm{n}$-order differential equations dependent on all derivatives," Applied Mathematics Letters, vol. 18, no. 2, pp. 209-218, 2005.

[22] P. H. Rabinowitz, "Some global results for nonlinear eigenvalue problems," Journal of Functional Analysis, vol. 7, no. 3, pp. 487$513,1971$. 


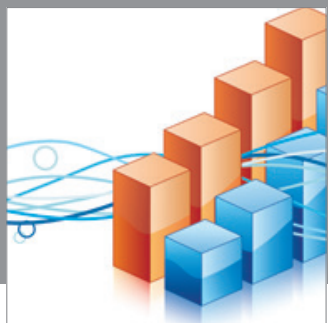

Advances in

Operations Research

mansans

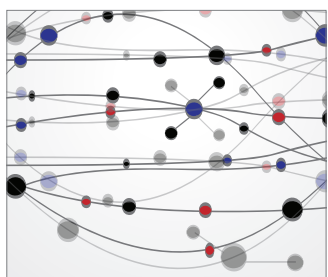

The Scientific World Journal
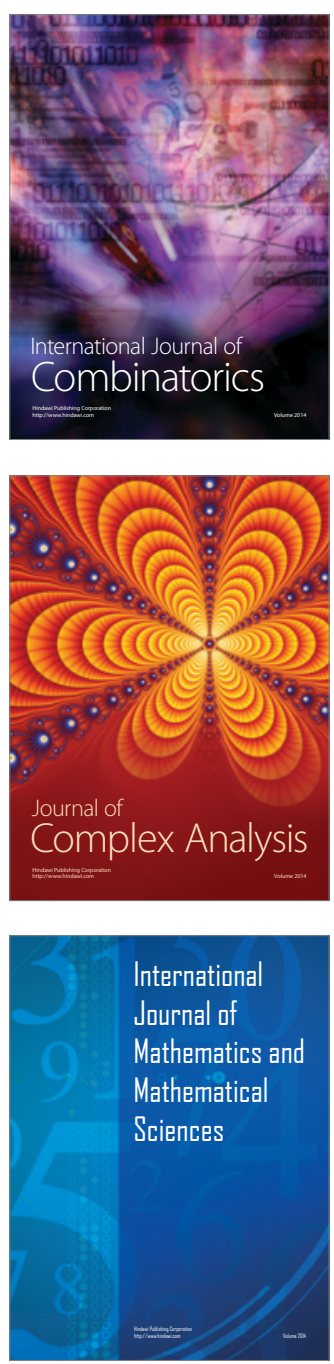
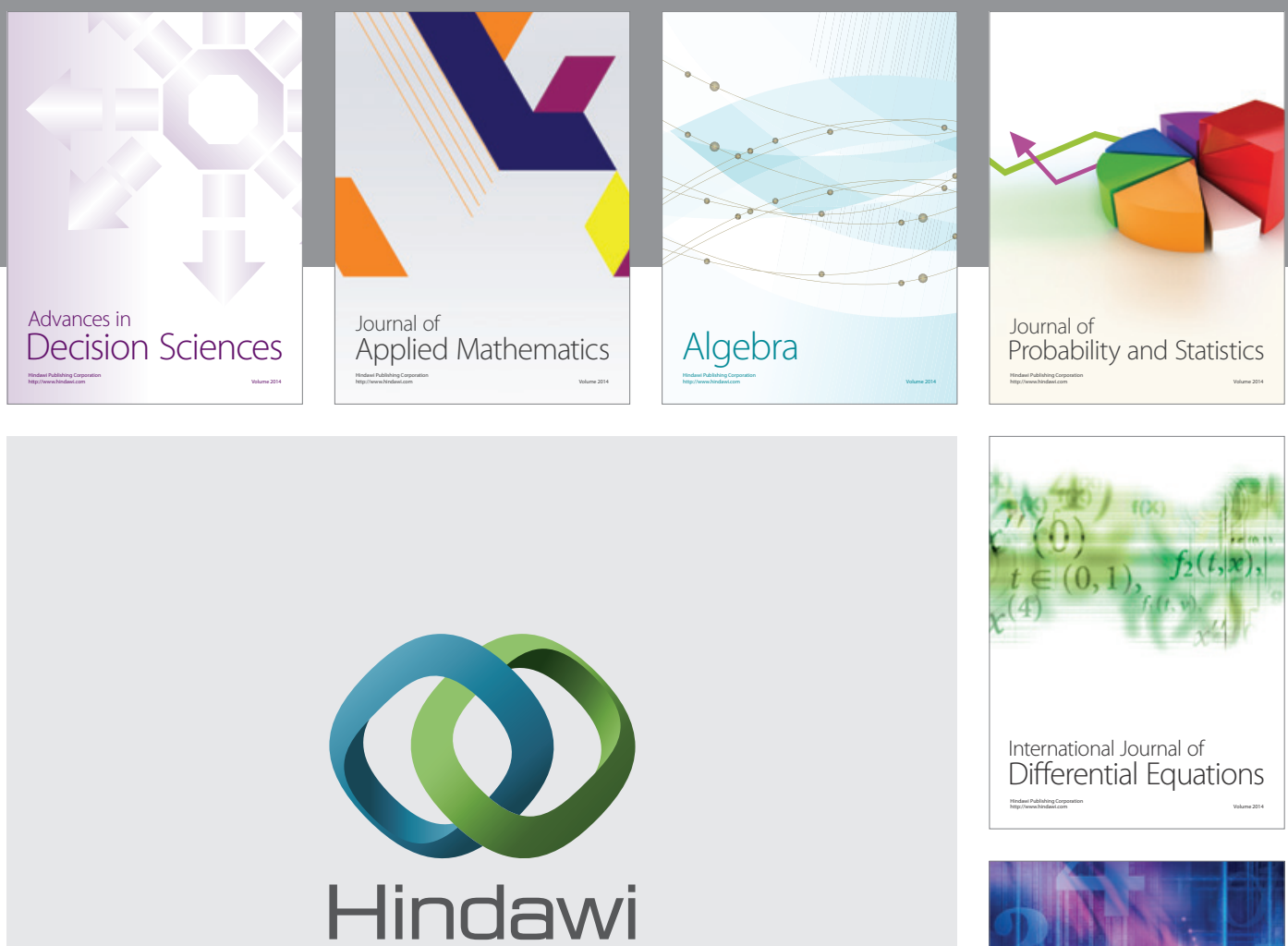

Submit your manuscripts at http://www.hindawi.com
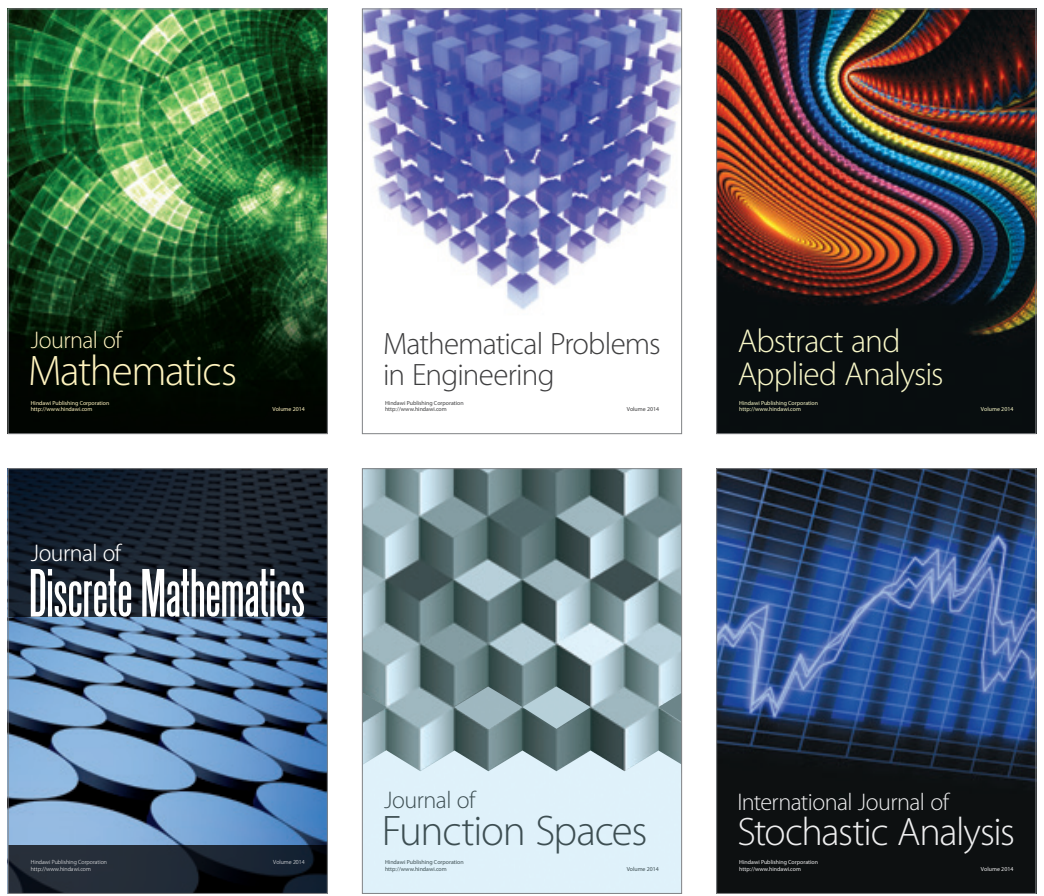

Journal of

Function Spaces

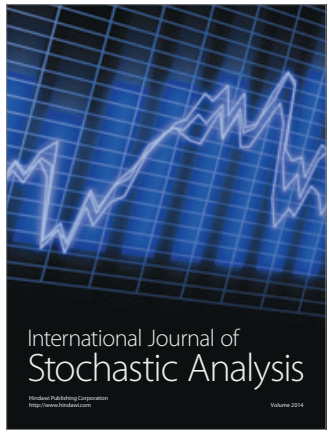

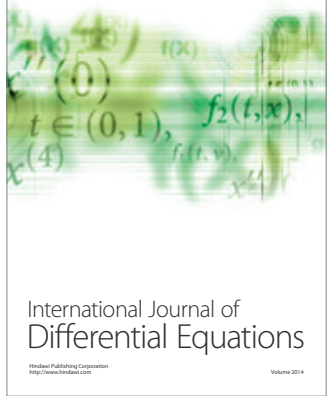
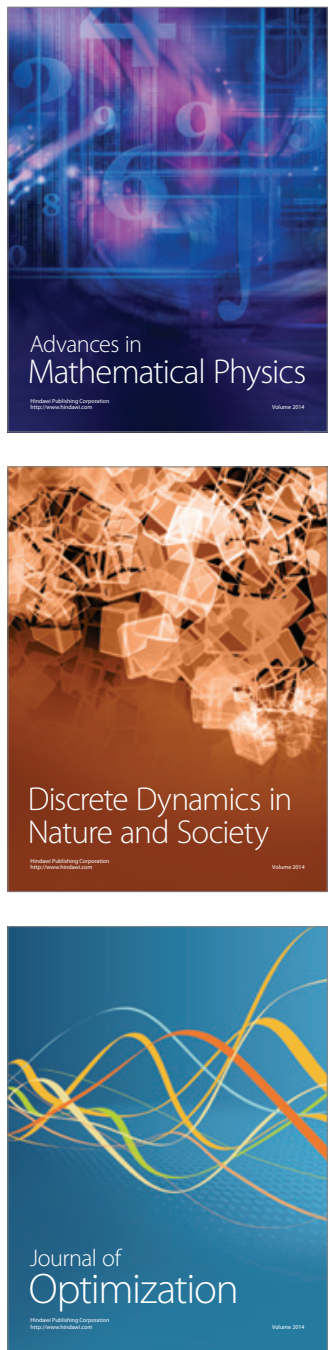